\title{
Resistance to extinction in rats as a function of drive level and amount of training
}

JOHN H. MUELLER, University of Missouri, Columbia, Mo. 65201, and D. GENE DAVENPORT, Saint Louis University, St. Louis, Mo. 63103

Forty-five rats were given 20, 50, or 100 training trials in a runway at $90 \%, 80 \%$, or $70 \%$ of base weight. Acquisition running speed was a monotonic increasing function of both training and drive levels. Alley speed over 25 extinction trials was nonmonotonically related to amount of training, with the 50-trial groups more resistant to extinction than 20 or 100 at all drive levels, and was inversely related to drive level. Time spent at the cup during both acquisition and extinction was a monotonic increasing function of both training and drive.

The relationships between resistance to extinction (RE) and various runway parameters have been reviewed by Sperling (1965), and the acquisition-reward magnitude and amount of training functions are of particular relevance here. When the magnitude of reward and the number of training trials were factorially manipulated, Ison \& Cook (1964) found that RE was less after $37-\mathrm{mg}$ rewards than after 370-mg rewards when 31 acquisition trials were used, but that the reverse was true when training was extended to 76 trials. Some studies have not found such a complete reversal with extended training (e.g., Hill \& Wallace, 1967), and other research (Clifford, 1964, 1968) has shown a nonmonotonic effect of amount of training, with RE increasing at first as training increases but then decreasing as training is further extended. A crucial factor seems to be the reward magnitude, although runway length may also be important, and perhaps some factor related to both.

The effect of drive level (D) on RE has been less explored in a comparable manner in the runway, since most such studies have either shifted to a new D in extinction or have not varied training within the same investigation. A proposal by Black (1965) that $\mathrm{D}$ contributes to incentive motivation through the consummatory goal response leads to the expectation that the amount of training and $D$ might affect $R E$ in the same way that reward and training seem to. Birch (1961) also suggested this possibility through considerations from frustration theory but noted that manipulation of $D$ through hours of deprivation has complications which make the effect difficult to study. D would also seem a potential variable on empirical grounds and a possible source of confounding between studies. The present study investigates the effect of $D$ at different levels of training, providing information about another combination of variables which is theoretically capable of influencing $R E$ via the frustration mechanism. Time spent at the food cup was used to supplement running measures as a more direct indicant of the magnitude of the goal response to determine its relationship to $\mathrm{D}$, training, and the instrumental response.

\section{SUBJECTS}

Forty-five female albino rats obtained from the St. Louis University Medical School colony, when about 60-days old, were randomly assigned to the nine groups.

\section{APPARATUS}

A 33-in. Hunter 380 nunway with 12-in. end boxes was used. The startbox and alley had Masonite flooring, while the goalbox had a grid floor. Running times were taken to the nearest $0.01 \mathrm{sec}$ in three alley segments: start time from the startbox door to a photobeam 4 in. outside the door, alley time from that beam to another 4 in. outside the goalbox door ( 25 in.), and entry time from 4 in. outside the goalbox to a beam over the food cup. The beam terminating entry time was also wired to run a fourth clock cumulatively whenever the $\mathrm{S}$ was over the food cup; the beam passed $3 / 8$ in. above and $1 / 2$ in. behind the front lip of the cup. This measure was considered to reflect the vigor of the consummatory goal response during the fixed period of goal confinement.

\section{PROCEDURE}

Three levels of training and drive were used in a factorial arrangement: 20,50 , or 100 training trials at $90 \%, 80 \%$, or $70 \%$ of base weights as drive levels. Base weights were established over 6 days prior to runway training, with $S s$ then reduced to the proper levels and given two brief sessions in the open runway for magazine training. All Ss were reduced at the same time, so that the drive levels had been in effect for the same period of time in each training group, although training groups did not begin on the same day.

The Ss were run in groups of three, with each given one trial in sequence, yielding an intertrial interval of about $2 \mathrm{~min}$. Ten trials per day were given, with $1045-\mathrm{mg}$ pellets as a reward on each trial. Training groups were staggered in starting so that all began extinction on the same day, with Ss not yet started given a short period of handling daily. When Ss failed to leave the startbox within $60 \mathrm{sec}$, they were nudged into the alley and similarly given $60 \mathrm{sec}$ to enter the goalbox or nudged in. All Ss were confined in the goalbox for $30 \mathrm{sec}$ on each trial. After 20,50 , or 100 trials on the immediately preceding days, the Ss were given 25 extinction trials, 10 on the first 2 days, and 5 on the third day.

\section{RESULTS}

The first 20 training trials were analyzed, using reciprocals of the running measures, and total time spent at the cup, as data in 3 by 3 by 20 repeated-measures analyses of variance. The dummy main effect (total training to be given) produced no significant effects, indicating initial comparability, although the expected drive effect did occur for start, alley, and entry speeds, and cup time, $F s(2,36)=3.56$, $8.24,8.64$, and 9.85 , respectively, $\mathrm{p}<.05$, with high-drive groups running faster and spending more time at the cup, with no Training by Drive interactions.

An identical analysis was performed for the final 20 trials, using the same trials for the 20-trial groups. These indicated quite significant $(\mathrm{p}<.001)$ training and drive effects, with high levels of both producing faster running and more time at the cup. Training by Drive interactions resulted for start and alley speeds, $F s(4,36)=3.50$ and $3.18, \mathrm{p}<.05$, as the drive subgroups separated more after 50 and 100 trials than with 20 , but this was not the case for entry speed or cup time.

Each of the four measures in extinction was analyzed using a 3 by 3 by 25 repeated-measures analysis of variance. Figure 1 presents the alley-speed means for blocks of five trials for each drive level at each training level for the final acquisition block and the five extinction blocks, with five Ss per block or a total of 25 observations per mean. The nature of the effects seems clear: the 50-trial groups were more resistant to extinction than were the 20- or 100-trial groups, and the high-drive groups were the least resistant to extinction. The alley-speed analysis indicated a significant training effect, $F(2,36)=6.21, p<.01$, but no drive effect $(\mathrm{F}<1)$, and no Training by Drive interaction $(F<1)$. The complete reversal in drive levels from acquisition order produced a Drive by Trials interaction in extinction, $F(48,864)=2.26, p<.01$. The Training by $T$ rials interaction was also significant, $F(48,864)=1.57, p<.01$, as the 50-trial groups did not extinguish as rapidly as did the 20 and 100 . This trend apparently started first at the low-drive level, but the triple interaction was not significant $(F<1)$. Since the trends in 


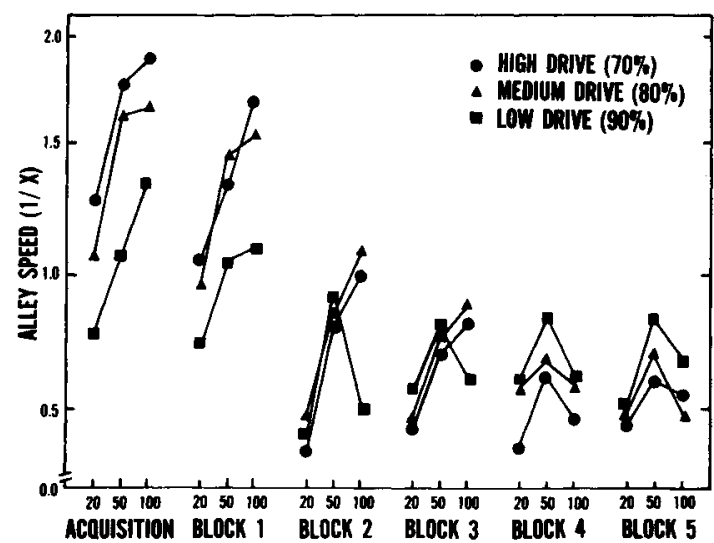

Fig. 1. Alley speed for each drive and training level at the end of acquisition and over extinction blocks.

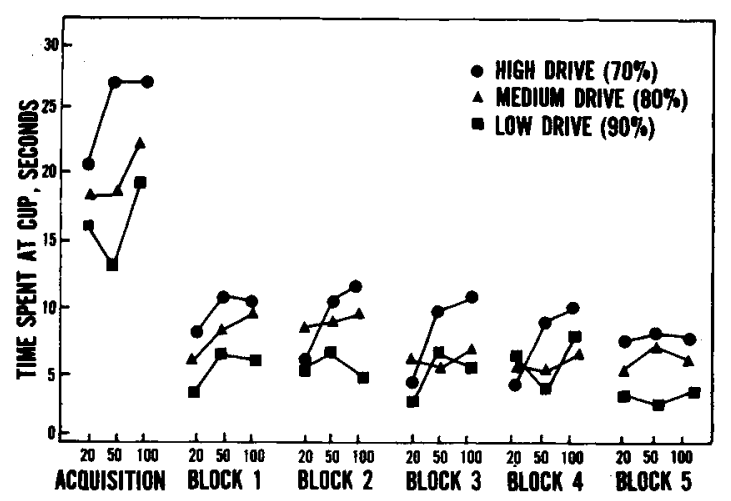

Fig. 2. Time spent at the cup for each drive and training level at the end of acquisition and over extinction blocks.
Blocks 4 and 5 of Fig. 1 are in the opposite direction from acquisition, "correction" for the acquisition differences would basically only accentuate the effects; an attempt to apply Anderson's (1963) shape-function analysis was unsuccessful due to the trial-to-trial variability.

The entry-speed analysis indicated that the nonmonotonic effect due to training was limited to the $80 \%$ and $70 \%$ groups, and the reversal of drive levels did not occur. The cup-time data indicated quite different RE effects and are presented in Fig. 2. The monotonic increasing trend that developed during acquisition seemed to remain, in general, unlike alley speed and the higher-drive levels of entry speed. Although this monotonic relationship is not ideal, the differences in the trends in Fig. 1 and Fig. 2 seem quite clear. The training and drive main effects were significant, $F s(2,36)=3.37$ and 6.04 , $\mathrm{p}<.01$, with high levels of each producing more time at the cup, with no interaction.

\section{DISCUSSION}

All four measures revealed similar acquisition trends, a monotonic increasing function relating the measures to the amount of training and $D$ level. With the exception of start speed, the measures also revealed specific enduring RE patterns, although of a different nature. Alley speed indicated a nonmonotonic relationship between speed and training level, such that RE increased as training went from 20 to 50 trials then decreased as training was further extended to 100 trials. A similar trend was found for the high and middle drive levels of entry speed. On the other hand, cup time maintained the monotonic increasing trend between time and training and $D$ level for as long as any relationship remained in extinction. While this might suggest that measures taken farther from the goal show less monotonicity with regard to the RE training function, that is a difficult comparison and is only suggested by the data.

The expectation that $\mathrm{D}$ and training might interact as reward magnitude and training have been shown to do in determining RE (Ison \& Cook, 1964) is not supported in the present data, although this might be a result of procedural features. For example, a smaller reward magnitude or a common extinction drive might produce such a result. The present data seem quite clear on the RE-training function, however, with a nonmonotonic relationship for the running measures, in a short runway, with fairly large reward and over a wide range of $D$ levels.

The RE-D function is mixed in the present data (inverse for alley speed but direct for entry speed and cup time), suggesting a need to distinguish $R E$ measures, since the same definition of $D$ was used throughout. The present data might suggest that the more the measure used reflects the strength of the consummatory response rather than the instrumental response, the more direct the
RE-D relationship (viz, cup time), while the reverse results in an inverse relationship. Thus short runways or Skinner boxes might yield response measures which largely reflect consummatory or immediate approach components more than would the longer runway. This speculation might draw upon a number of "gradient" constructs as mechanisms, but research directed at this specific question is needed for clarification. REFERENCES

ANDERSON, N. H. Comparison of different populations: Resistance to extinction and transfer. Psychological Review, 1963, 70, 162-179.

BLACK, R. W. On the combination of drive and incentive motivation. Psychological Review, $1965,72,310-317$.

BIRCH, D. A motivational interpretation of extinction. In M. R. Jones (Ed.), Nebraska Symposium on Motivation: 1961. Lincoln: University of Nebraska Press, 1961. Pp. 179-197.

CLIFFORD, T. Extinction following continuous reward and latent extinction. Journal of Experimental Psychology, 1964, 68, 456-465. CLIFFORD, T. Runway length and the failure of expected rewards: the OEE. Canadian Journal of Psychology, 1968, 22, 417-426.

HILL, W. F., \& WALLACE, W. P. Reward magnitude and number of training trials as joint factors in extinction. Psychonomic Science, 1967, 7, 267-268.

ISON, J. R., \& COOK, P. E. Extinction performance as a function of incentive magnitude and number of acquisition trials. Psychonomic Science, 1964, 1, 245-246.

SPERLING, S. E. Reversal learning and resistance to extinction: A review of the rat literature. Psychological Bulletin, 1965, 63, 281-297. 